\title{
Dual polarized traveling wave antenna for Ultra Wideband radar application
}

\author{
Grzegorz Adamiuk*, Christian Sturm*, Thomas Zwick* and Werner Wiesbeck* \\ *Institut für Höchstfrequenztechnik und Elektronik \\ Universität Karlsruhe (TH) \\ Kaiserstr. 12, 76128, Karlsruhe, Germany \\ email: grzegorz.adamiuk@ihe.uka.de
}

\begin{abstract}
This paper introduces a development of a compact dual polarized traveling wave antenna for Ultra Wideband technique. A principle of the antenna is introduced as well as its development. Subsequently the measurements results are shown including the time domain analysis of the antenna behavior.
\end{abstract}

\section{Introduction}

Bandwidth of the signal is the main factor that can directly enhance a resolution of a radar system. Therefore UWB has become very important research and development issue in the whole world. With the bandwidth of $7.5 \mathrm{GHz}$ allowed in the USA by the FCC [1] or $2.5 \mathrm{GHz}$ allocated in the Europe [2] UWB has a big potential in remote sensing with very high resolution. Due to dispersion of the signal and low power density allowed for a usage, a main application of such a device is close range remote sensing e.g. indoor radar, through-wall radar or GPR. The performance of such a system can be improved by evaluation of the polarization properties of the object. Since there exist almost no dual polarized antenna elements with convenient time domain behavior suitable for UWB technique and common for both polarizations phase center of radiation, the focus was put on the development of such devices.

\section{Construction of the antenna}

The proposed antenna belongs to the group of dielectric rod antennas, which radiate electromagnetic wave by a traveling wave principle. In the literature can be found some dual polarized dielectric rod antennas, which require a complicated and bulky out of phase feeding networks [3]. The proposed antenna (see Fig.1) is a small size dual polarized antenna with bandwidth suitable for UWB applications. It consists of clearly separated feeding network, launcher and a radiating element. The feeding network is build of two aperture coupled baluns, which are positioned orthogonally to each other and transform the microstrip lines to the two orthogonally oriented slot lines. It is etched on the RO5880 substrate with $\varepsilon_{\mathrm{r}}=2.2$. Further the slots are galvanically connected to the launcher, which is made of self-adhesive cooper foil. As a carrier for the launcher serves a dielectric material that also the radiating

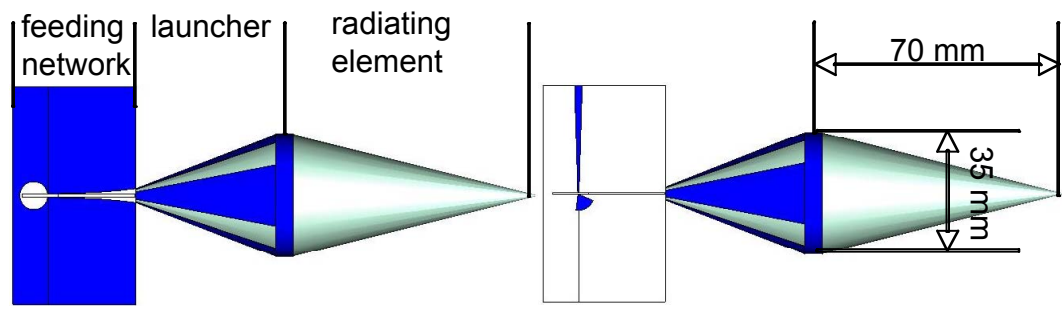

left view right view

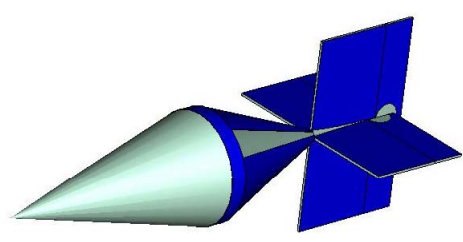

3D view

Fig.1 Model of the antenna in the CST Microwave Studio 
section is built of. As a dielectric material the Teflon with relative dielectric permittivity of 2.08 was used. Teflon has very good mechanical properties and very low dielectric losses, which prevent a dissipation of the energy in the antenna. The radiating element is formed to the dielectric cone. It enables a smooth transition of the wave into the air and forms the radiation pattern. Such a configuration led to the development of very compact dual polarized UWB antenna. A small dimension in the transversal direction (diameter of the cone $35 \mathrm{~mm}$ ) allows for future application of the antenna to the ultra broadband antenna array, where a small distance between elements is of big importance. The realized antenna is shown in Fig.2.

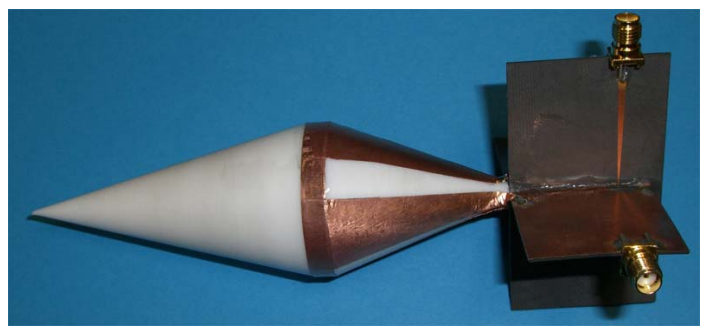

Fig.2 Realization of the dual polarized ultra broadband dielectric rod antenna.

The electric field distribution in the antenna and its near field at three different frequencies was simulated in the CST Microwave Studio. The results are depicted in Fig.3. A clear directive radiating performance of the antenna can be observed. A stronger spurious radiation with increasing frequency can be observed. It is caused by relatively high mechanical complexity of the whole object. Each transition (microstrip line-slot line, slot line-launcher, launcher-radiating section) causes an undesired radiation. However most of the energy is radiated in the intended direction, which will be presented in the next parts of the paper.

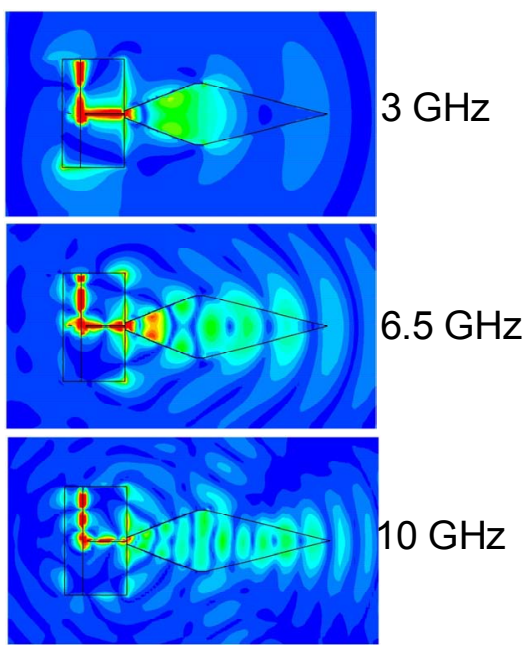

Fig.3 Field distribution in the antenna in the E-Plane at three frequencies

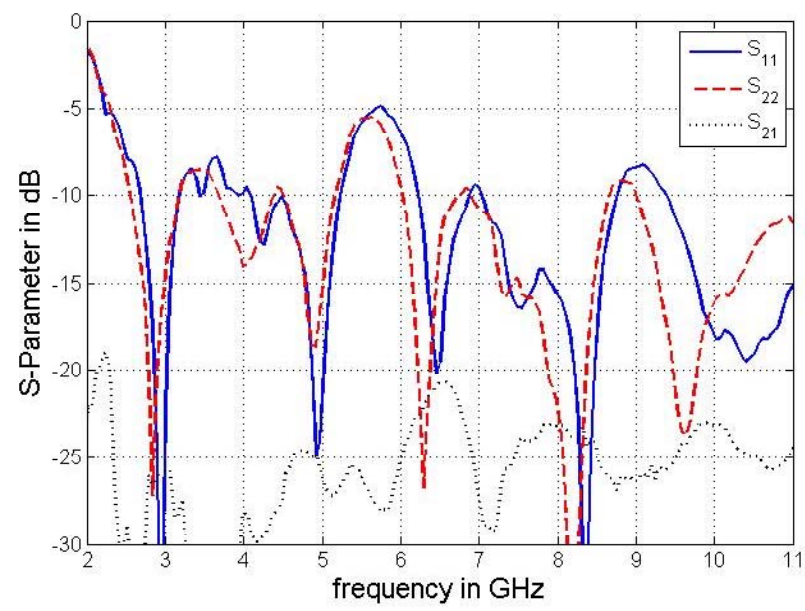

Fig.4 Measured S-Parameters (matching and coupling) at two ports of the antenna

\section{Measurement results}

The measured S-parameters of the realized antenna are depicted in Fig.4. It can be observed that the antenna has an acceptable matching in the frequency range above approx. $2.5 \mathrm{GHz}$. Furthermore both ports show similar matching performance, which indicates in the case of this particular device similar far field properties for both polarizations. In the frequency range between approx. $5.2 \mathrm{GHz}$ and $6.2 \mathrm{GHz}$ the matching reaches the level of $-5 \mathrm{~dB}$. This has however only minor influence on the overall antenna performance, which is designated for the pulse mode operation with the bandwidth exceeding $7 \mathrm{GHz}$. In other words, due to worse 

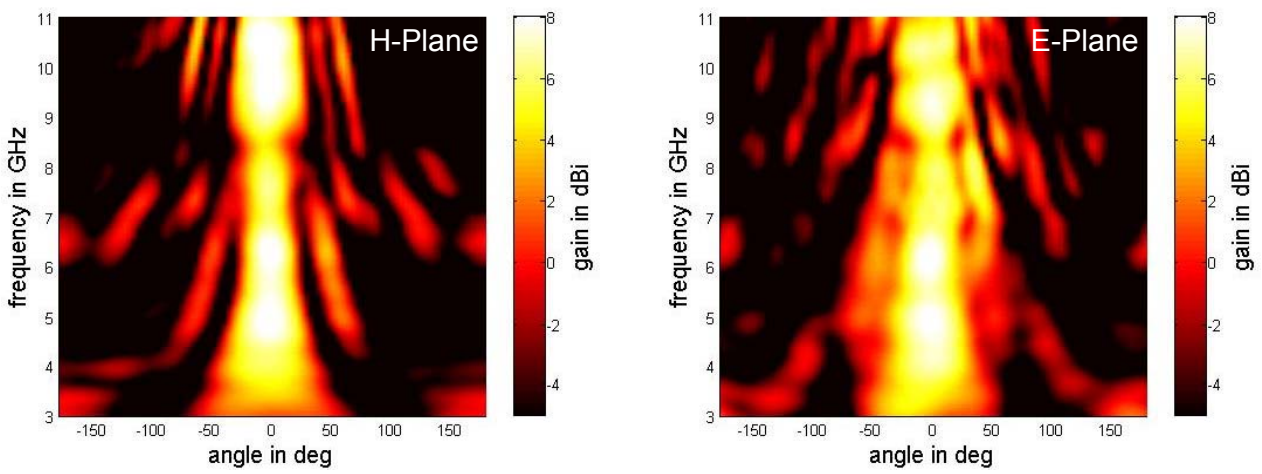

Fig.5 Measured gain of the antenna for one polarization in the H-Plane and E-Plane (Co-Pol)

matching in the particular frequency range no significant influence on the antenna impulse response was noticed. The measured gain in the H-Plane and E-Plane for Co-Polarization from $3 \mathrm{GHz}$ to $11 \mathrm{GHz}$ can be seen in Fig.5. It can be noticed, that the antenna radiates for each frequency with similar properties. The beam is relatively concentrated and maximal achieved gain is higher than $8 \mathrm{dBi}$. The side lobes are present as typical for that kind of antennas, but due to their weakness can be neglected. A high concentration of the radiated energy in a particular direction can allow for the penetration of the UWB signals through the wall or other lossy objects. The proposed design allows for further development of the broadband antenna arrays, which enhance the gain. By application of e.g. four elements the maximal gain can reach even $14 \mathrm{dBi}$, which could be sufficient to "look" with UWB signals through typical walls.

Evaluating dual polarized antennas the polarization purity in the far field of the antenna has to be taken into account. For this purpose the gain of the antenna in the cross polarized configuration was measured in the anechoic chamber. The results of the measurements of the cross polarized gain over frequency for H-Plane and E-Plane is shown in Fig.6. A clear decoupling of radiated polarizations can be noticed. It can be observed that for most frequencies and angles the cross polarized components are weaker than $-12 \mathrm{dBi}$. For a better impression of the polarization decoupling the gain for $\mathrm{Co}$ - and X-Polarization in the main beam direction is shown in Fig.7. In the whole frequency range the decoupling of polarizations is better in average than $15 \mathrm{~dB}$. It means that the cross polarized components are weaker than $5 \%$ of the co-polarized ones, which allows for clear definition of the polarization in the far field.

For the evaluation of the performance of the ultra broadband antennas for puls mode operation a traditional frequency domain description by gain and radiation pattern is not
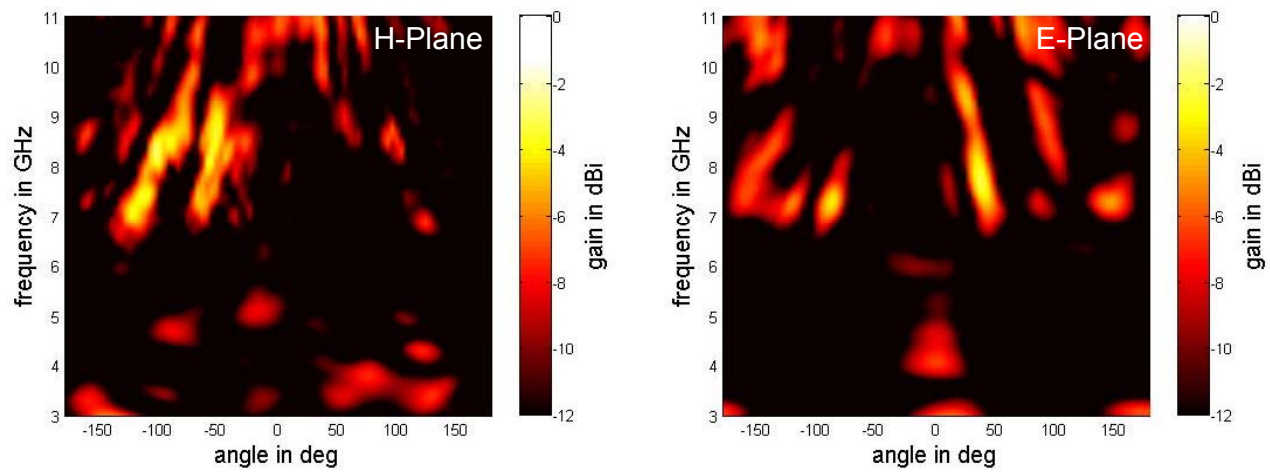

Fig.6 Measured gain of the antenna for one polarization in the H-Plane and E-Plane (X-Pol) 
sufficient. In order to investigate the distorting properties of the antenna a phase response in the frequency domain or impulse response in the time domain have to be included in the description. For this purpose the complex transfer function of the antenna was measured in the anechoic chamber in the frequency domain. Application of the Fourier transform allowed for calculation of the impulse response of the antenna [4,5], which is presented in Fig.8.

As can be noticed the impulse response of the antenna in the main beam direction is very short. It means that the pulse fed to the antenna does not change significantly its form during propagation through the device. It can be also noticed that in spite of worse matching in the particular frequency range the time domain behavior is not significantly affected by this fact. The antenna can be successfully applied for the broadband time domain operation with UWB systems.

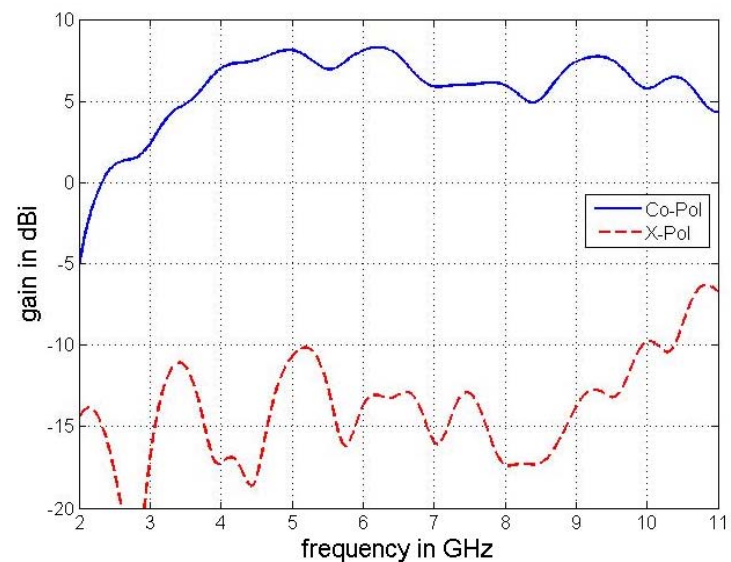

Fig.7 Measured gain of the antenna in the main beam direction for Co- and X-Pol

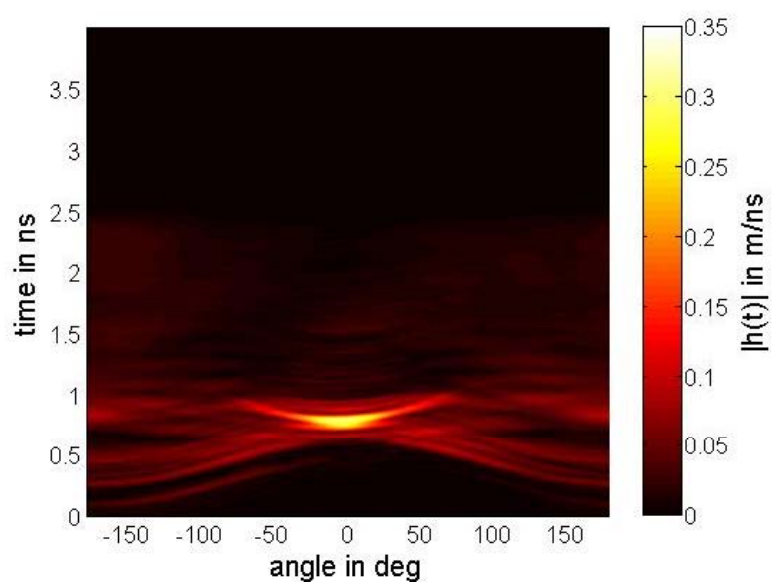

Fig.8 Impulse response of the antenna for one polarization in the H-Plane (Co-Pol)

\section{Conclusions}

The designed antenna is a promising candidate for high-end, close range UWB radar systems. Since there exist almost no dual polarized UWB antenna elements, the proposed device can be an important issue in a further research comprising polarimetric UWB systems. Additionally small dimension in transverse direction allow for the realization of high gain UWB antenna arrays, which are required in e.g. through wall radar or GPR.

\section{Reference:}

[1] Federal Communications Commission (FCC), Revision of Part 15 of the Commission's Rules Regarding Ultra Wideband Transmission Systems, First Report and Order, ET Docket 98-153, FCC 0248; Adopted: February 14, 2002; Released: April 22, 2002.

[2] The Commission of the Eurpean Communities, "Commission decision on allowing the use of the radio spectrum for equipment using ultra-wideband technology in a harmonised manner in the Community", Official Journal of the European Union, 21 February 2007.

[3] Jae-Young Chung, Chi-Chih Chen „Ultra-wide bandwidth two-layer dielectric rod antenna”, Antennas and Propagation International Symposium, 2007 IEEE, Page(s):4889 - 4892, 9-15 June 2007

[4] Sörgel, W., Waldschmidt, C., Wiesbeck, W.; "Transient responses of a Vivaldi antenna and a logarithmic periodic dipole array for ultra wideband communication", Antennas and Propagation Society International Symposium, 2003. IEEE Volume 3, Page(s):592 - 595, 22-27 June 2003

[5] W. Sörgel and W. Wiesbeck, "Influence of the Antennas on the Ultra Wideband Transmission", EURASIP Journal on Applied Signal Processing, special issue UWB - State of the Art, pp. 296-305, March 2005 